\title{
Residual rightward attentional bias after apparent recovery from right hemisphere damage: implications for a multicomponent model of neglect
}

\author{
Jason B Mattingley, John L Bradshaw, Judy A Bradshaw, Norman C Nettleton
}

\begin{abstract}
Unilateral neglect may be a multicomponent attentional disorder consisting of an initial automatic orienting of attention toward the ipsilesional side and a subsequent impairment in contralesionally reorienting attention, both of which are superimposed on a generalised reduction in attention resources. It has been hypothesised that patients' ability to reorient attention contralesionally may recover relatively quickly, but that the ipsilesional attention bias may be relatively persistent. This hypothesis was tested by consecutively examining 13 patients who had had a right hemisphere stroke, and who had left unilateral neglect. They were examined once shortly after the stroke and again 12 months later, using a battery of standard clinical and experimental tasks. Patients initially showed a strong and consistent rightward attentional bias in addition to a failure to reorient their attention leftward. After 12 months patients continued to show an abnormal ipsilesional attentional bias, though most were now able to fully reorient their attention toward the contralesional side. These results suggest that restitution of the capacity to reorient attention contralesionally may underlie the apparent recovery from clinical signs of unilateral neglect. The presence of a residual ipsilesional attentional bias in most patients, however, may, at least in part, account for the poor functional outcomes in some apparently "recovered" patients.
\end{abstract}

$(\Im$ Neurol Neurosurg Psychiatry 1994;57:597-604)

Unilateral neglect is a disorder of spacerelated behaviour which is a common concomitant of unilateral (particularly right) hemispheric damage ${ }^{1-5}$ (though see Ogden) ${ }^{67}$ The behavioural manifestations of neglect in the acute stages after injury are well documented, ${ }^{1}$-10 though relatively few studies have assessed patients in the "chronic" stage(s) of recovery. ${ }^{11-15}$ Thus, although there are several models purporting to account for the impairments shown by patients with unilateral neglect, ${ }^{816-20}$ few have considered the potential implications for such models of subsequent functional recovery in patients who currently show acute unilateral neglect. The purpose of this study was to evaluate the extent of recovery from the deficits which characterise neglect on a variety of clinical and experimental tasks.

Many workers, ${ }^{18-21}$ though not all, ${ }^{16}$ have proposed that neglect is due to a disruption of normal attentional processes subserved by single or multiple neural circuits. ${ }^{19} 22$ One of the most influential of these models, that proposed by Posner and coworkers, ${ }^{21}{ }^{23}$ assumes the existence of three separate operations involved in the voluntary orienting of spatial attention (that is, disengage, move, and engage). It has been suggested that any of these operations may be selectively disrupted by lesions of specific brain structures. ${ }^{24}$ Of particular relevance to the consideration of neglect, it has been shown that patients with parietal lesions are impaired in "disengaging" their attention from its present focus in preparation for a shift toward the contralesional side. $^{21}{ }^{23}$ Reinstatement of the capacity to effectively reorient attention toward the contralesional side may therefore underlie the progressive recovery from neglect which has been so widely reported..$^{14}$ 25-27 Moreover, at least one study has found convincing evidence that, with appropriate long-term intervention, patients with left neglect can overcome their difficulty in directing attention contralesionally. ${ }^{28}$

Unfortunately, the model of Posner and coworkers can only account for a subset of the behavioural manifestations of unilateral neglect. There is ample evidence to suggest that neglect may also be characterised by a relatively early, "automatic" (perhaps obligatory) orienting of attention to stimuli on the ipsilesional side. ${ }^{29}{ }^{30}$ Indeed, the phenomenon of "magnetic gaze attraction" 30 or "peeking" 31 toward the ipsilesional stimulus on confrontation testing of the visual fields has long been known by clinicians. In fact, this phenomenon may simply be a more florid manifestation of the tendency among patients with neglect to orient their attention (either overtly or covertly) to stimuli on the ipsilesional side. ${ }^{32}$ Indeed, several early studies ${ }^{233}{ }^{34}$ documented the tendency of patients with right hemisphere damage to adopt a right-sided "position preference" when selecting stimuli (for example, in Raven's matrices) from an array of response alternatives, even in the absence of any other signs of left neglect.

Subsequent investigations ${ }^{13} 173032$ have supported the notion of an early, automatic orienting of attention toward the ipsilesional side. In one study, ${ }^{32}$ left hemisphere and right hemisphere damaged patients were tested on 
an overlapping figures task. It was found that patients with right hemisphere damage, even those without clinically manifest neglect, tended to first report figures on the ipsilesional side of the stimulus, unlike patients with left hemisphere lesions and controls, who tended to report first figures on the left. Such findings have led to the formulation of a multicomponent model of neglect. ${ }^{132}$ This model assumes that there are (at least) three separate deficits which characterise neglect: (a) an initial, automatic orienting of attention toward the ipsilesional side; (b) an impairment in disengaging attention from the ipsilesional side and reorienting attention toward stimuli on the contralesional side; and (c) a generalised (that is, directionally non-specific) reduction in attentional/information processing capacity. ${ }^{35-37}$

One important distinction between the two direction-specific components of this model is their temporal ordering. Thus, the automatic ipsilesional orienting occurs first, immediately
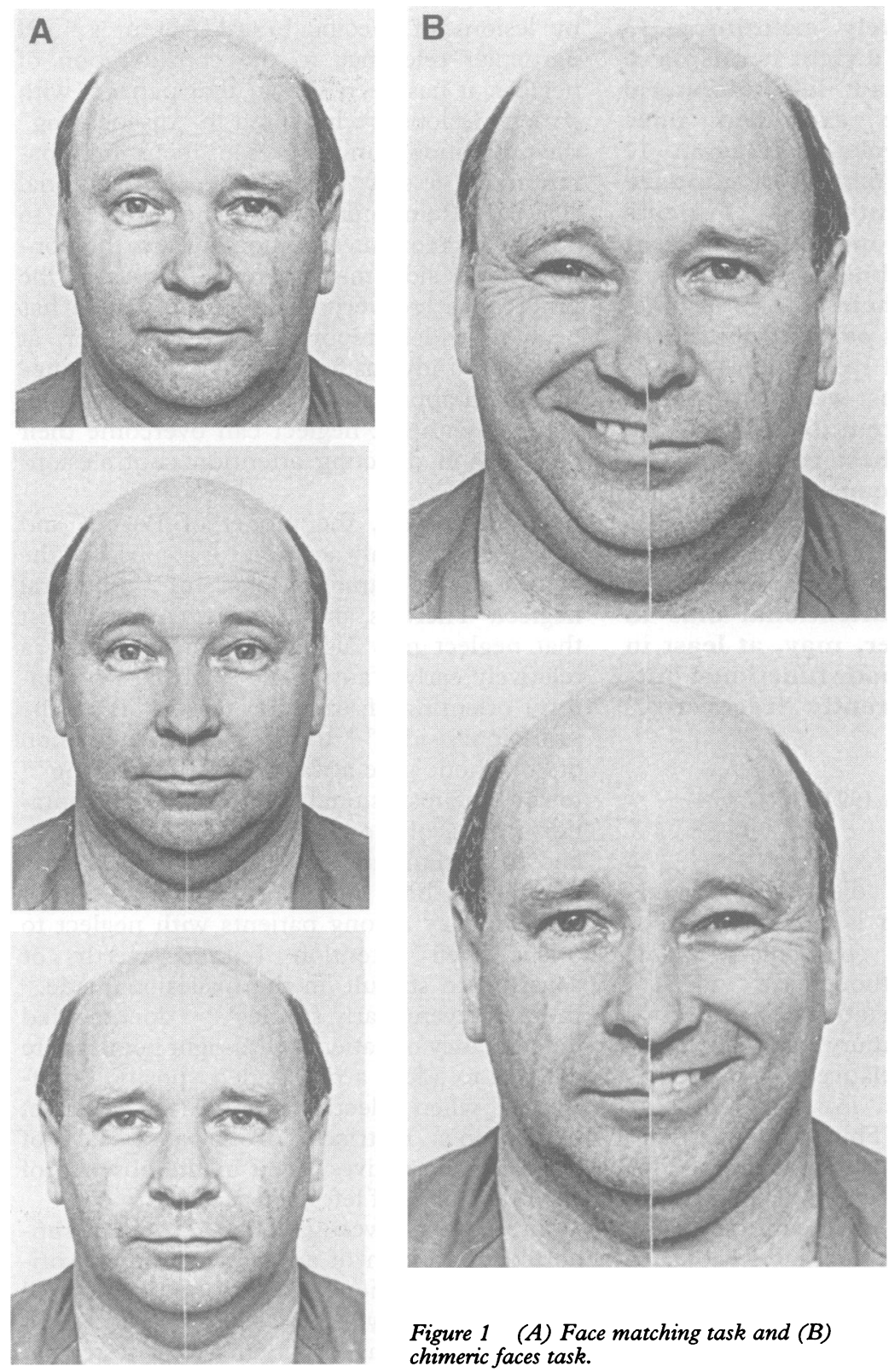

Figure 1 (A) Face matching task and (B) chimeric faces task. on activation of the visual attentional system, whereas operations involved in shifting (or reorienting) attention occur after this initial orienting. ${ }^{38}$ Thus the occurrence of unilateral neglect reflects an initial automatic orienting toward the ipsilesional side, and a subsequent failure to reorient attention contralesionally. Several studies, using different paradigms, have confirmed the dissociability of these two attentional operations. ${ }^{23} 303839$

Karnath $^{13}$ proposed that the second component (that is, the capacity to reorient toward the contralesional side) recovers faster than the other two, such that among patients in whom the more familiar signs of neglect have recovered, there may nevertheless be a persistent attentional bias toward the ipsilesional side. Following cognitive rehabilitation, Pizzamiglio et $a l^{40}$ documented significant improvements in patients with left neglect on tasks of visual exploration and scanning, but observed relatively small improvements on a measure of perceptual bias. Thus, whereas patients with right hemisphere damage may initially show both of the direction specific components of neglect, they may subsequently show only the ipsilesional attentional bias without a deficit in their ability to redirect attention contralesionally. The aim of our study was to test this prediction by examining a group of patients with left neglect on tests which measure, respectively, automatic attentional bias for visual stimuli and the ability to reorient attention contralesionally across peripersonal space. Patients were first tested soon after their stroke and subsequently after an interval of 12 months.

To quantify patients' ability to redirect attention toward and into contralesional space, we used a battery of cancellation tasks and a line bisection test. Although there is evidence for dissociation, at an individual level, between impaired and normal performance on these two types of task, ${ }^{41}$ several group studies have confirmed that they are highly intercorrelated ${ }^{42}$ and in fact measure a single ability, namely, visual attentional scanning. ${ }^{43}$ We also administered two tests which measure opposing visual attentional biases in normal subjects and in those with left unilateral neglect. ${ }^{44}$ In the face matching task subjects were required to indicate which of two bisymmetrical composites (one composed of the two left halves of the poser's face, the other composed of the two right halves) more closely resembled the original (inherently asymmetrical) photograph (see fig 1A). In the chimeric faces task subjects were required to judge which was the "happier" of a pair of chimeric face stimuli, one the mirror image of the other. In one face, the smiling half was on the left, in the other the smiling half was on the right (see fig 1B). Using similar tasks, several previous studies have shown the existence in normal adults of a consistent left sided attentional bias. ${ }^{45} 46$ In contrast, we documented a strong and consistent rightward attentional bias in patients (tested soon after their stroke) with right hemisphere damage and clinical signs of left unilateral neglect, 
though the same materials provided the expected pattern of a leftward bias with normal controls. ${ }^{44}$ The tasks used in this study were selected on the basis of their proved reliability and validity, ${ }^{46}$ their documented sensitivity to the deficits of interest, ${ }^{44}$ and because they could be attempted by all but the most severely impaired patients.

\section{Subjects and methods \\ SUBJECTS}

Thirteen patients with left unilateral neglect (eight men, five women) participated in the experiment. For 12 of these patients, an account of data obtained from their initial assessment on the two faces tasks has been presented elsewhere. ${ }^{44}$ The mean (SD) age of the patients at the time of their initial assessment was $64.4(9.7)$ years. All patients had unilateral right hemisphere lesions as inferred from clinical examination and confirmed by cranial CT scan. Table 1 gives the age, sex, and clinical details for each patient. Patients were screened for gaze disturbances before testing and visual fields were examined by confrontation testing. Handedness was assessed by a 10-item questionnaire which obtained information on familial handedness and personal hand preference for a variety of unimanual tasks. ${ }^{47}$ All subjects were assessed as being right-handed, and none had primary sensory or motor disturbances in this limb. Six patients were discharged from hospital without any formal rehabilitation (patients $4,5,9,10,11$, and 13 ), while the remaining seven patients received general occupational therapy and physiotherapy but no specific visual scanning or attentional retraining. Patients' functional capacity was determined using the Barthel activities of daily living index. ${ }^{48}$

\section{MATERIALS AND PROCEDURE}

Cancellation and line bisection tasks

An identical protocol was followed for the initial and 12 month follow-up assessments. Each patient was given a line cancellation task (Albert lines), ${ }^{49}$ a circle cancellation task, ${ }^{50}$ and the star cancellation task from the behav-

Table 1 Age, sex, and clinical details of patients with left unilateral neglect

\begin{tabular}{|c|c|c|c|c|c|c|}
\hline $\begin{array}{l}\text { Patients } \\
\text { No }\end{array}$ & Age/sex & Lesion * & $\begin{array}{l}\text { Time of initial } \\
\text { testing (days } \\
\text { since stroke) }\end{array}$ & $\begin{array}{l}\text { Visual } \\
\text { field } \\
\text { assessment * }\end{array}$ & Hemiplegia * & Barthel score* \\
\hline $\begin{array}{l}1 \\
2 \\
3 \\
4 \\
5 \\
6 \\
7 \\
8 \\
9 \\
10 \\
11 \\
12 \\
13\end{array}$ & $\begin{array}{l}67 / \mathrm{M} \\
72 / \mathrm{F} \\
43 / \mathrm{M} \\
60 / \mathrm{M} \\
80 / \mathrm{F} \\
60 / \mathrm{F} \\
66 / \mathrm{M} \\
68 / \mathrm{M} \\
52 / \mathrm{F} \\
66 / \mathrm{F} \\
72 / \mathrm{M} \\
59 / \mathrm{M} \\
72 / \mathrm{M}\end{array}$ & $\begin{array}{l}\text { S } \\
\text { TP } \\
\text { FP } \\
\text { OP } \\
\text { S } \\
\text { FPS } \\
\text { FP } \\
\text { FTS } \\
\text { TP } \\
\text { P } \\
\text { F } \\
\text { FP } \\
\text { FTS }\end{array}$ & $\begin{array}{r}10 \\
31 \\
34 \\
4 \\
4 \\
22 \\
423 \\
40 \\
9 \\
5 \\
13 \\
16 \\
24\end{array}$ & $\begin{array}{l}\text { LHH } \\
\text { NAD } \\
\text { NAD } \\
\text { LHH } \\
\text { NAD } \\
\text { NAD } \\
\text { NAD } \\
\text { LSQ } \\
\text { LHH } \\
\text { LHH } \\
\text { NAD } \\
\text { NAD } \\
\text { NAD }\end{array}$ & $\begin{array}{l}+++(++) \\
++(+) \\
++(+) \\
+(-) \\
++(-) \\
+(+) \\
+++(++) \\
++(++) \\
++(-) \\
+(-) \\
++(+) \\
+++(++) \\
+++(++)\end{array}$ & $\begin{array}{l}4(10) \\
10(20) \\
N C(17) \\
12(20) \\
9(20) \\
7(20) \\
5(7) \\
7(12) \\
17(20) \\
6(20) \\
\text { NC(20) } \\
7(14) \\
6(8)\end{array}$ \\
\hline
\end{tabular}

* $\mathrm{F}=$ frontal; $\mathrm{O}=$ occipital; $\mathrm{P}=$ parietal; $\mathrm{T}=$ temporal; $\mathrm{S}=$ subcortical; $\mathrm{LHH}=$ left homonymous hemianopia; $L \mathrm{SQ}=$ left superior quadrantanopia; $+++=$ severe; $++=$ moderate; $+=$ mild; $-=$ absent); Barthel score $=$ score $/ 20$ on Barthel activities of daily living index; $N C=$ month follow up.

Note: All patients, with the exception of patient 9, continued to show left peripheral visual field Note: All patients, with
loss after 12 months. ioural inattention test. ${ }^{51}$ These tasks, which form part of our standard protocol, ${ }^{44-52}$ were selected on the basis of their documented sensitivity to deficits of attentional reorienting. ${ }^{4243}$ Each sheet was placed directly in front of the patient and centred at the body midline. All patients used their preferred (ipsilesional) hand to hold the pen. Patients were also given a line bisection test consisting of 10 horizontal lines varying in length from 80 to $170 \mathrm{~mm}$ in $10 \mathrm{~mm}$ increments. These lines were centred on a single sheet of A4 paper in pseudorandom order and drawn through a mask with a central window, which prevented the patient from seeing previously or yet to be bisected lines. ${ }^{52}$ Errors were measured as the distance, to the nearest millimetre, between the patient's bisection mark and the true (objective) midpoint of the line. In accordance with established convention, errors to the right and left of the true midpoint were denoted as positive and negative respectively.

\section{Face matching task}

The method used to construct stimuli was based on that originally devised by Gilbert and Bakan. ${ }^{45}$ Each of 18 posers (nine men, nine women) was photographed with a neutral expression against a uniform white background. Six black and white prints were produced for each poser, three in normal orientation and three mirror-reversed. Left-left and right-right composites were made by dividing two of each of the prints in half along the midsagittal axis and recombining the halffaces to produce mirror-symmetrical composites. This produced four composite faces, each of which contained information from only one half of the original face. Stimulus cards were then constructed by mounting the remaining normal print (or mirror image) at the top of a piece of white card and arranging two different symmetrical composites below. The relative positions of the composite faces were counterbalanced such that each occupied the uppermost position for half of the items. Face stimuli measured $48 \times 60 \mathrm{~mm}$ and each pair was separated by a gap of about 2 $\mathrm{mm}$. Figure 1A shows an example of one of the stimulus items used in the experiment. The total set of 36 items was presented in the same order to all subjects, such that one item from each poser appeared in the first and second halves of the set.

\section{Chimeric faces task}

Construction of chimeric face stimuli was based on the method described by Levy et al. ${ }^{46}$ Each of ten posers (five men, five women) was photographed against a uniform white background. Each poser was photographed twice, once with a smiling and once with a neutral expression.

Four black and white photographs were printed for each poser, two in normal orientation and two mirror-reversed. Each pair consisted of one smiling and one neutral face. These photographs were then divided along the midsagittal axis and recombined to make two different chimeras. In one, the smiling 
half-face was on the left and the neutral halfface was on the right, and in the other this was reversed. Each of the two normal orientation chimeras was paired with its mirror image and rephotographed, once with the normal chimera at the top and the mirror image below, and once with the positions reversed. This resulted in a matched set of four pairs of chimeric stimuli from each poser, making a total of 40 stimulus pairs. Each chimeric face measured $59 \times 76 \mathrm{~mm}$. Figure $1 \mathrm{~B}$ shows an example of one of the stimulus cards used in the experiment. Chimeras from each poser appeared once, in pseudorandom order, within each set of 10 pairs.

The order of presentation of the faces tasks was counterbalanced across patients. A short break was given between each task. For viewing the face stimuli were placed in front of the patient, either sitting flat on a table surface or inclined slightly towards the viewer, at a distance of about $30 \mathrm{~cm}$. In the face matching task, subjects were asked to indicate which of the two composite faces more closely resembled the uppermost photograph. In the chimeric faces task, patients were asked to indicate which of the two faces looked happier. There was no time limit for completion on any of the tasks and patients were not provided with feedback about their performance.

\section{Scoring of faces tasks}

We calculated an asymmetry score to indicate the degree of perceptual bias shown by patients on each faces task. For any given item, a response was defined as left-biased if the patient selected the left-left composite or the "smile left" chimera (as viewed), and right-biased if the right-right composite or "smile right" chimera was selected. The asymmetry score was calculated as the number of items (face matching triplets or chimeric pairs) in which a rightward bias was shown, minus the number of items in which a leftward bias was shown, divided by the total number of items ( 36 for face matching task, 40 for chimeric faces task). Thus a significant negative score indicates a leftward bias, whereas a significant positive score indicates a rightward bias.

\section{Testing protocol}

Follow-up testing was conducted 12 months

Table 2 Percentage omissions on cancellation tasks and mean error (in $\mathrm{mm}$ ) on line bisection

\begin{tabular}{|c|c|c|c|c|c|c|c|c|}
\hline \multirow[b]{2}{*}{ Patient No } & \multicolumn{4}{|c|}{ Initial testing ${ }^{\star}$} & \multicolumn{4}{|c|}{12 months follow up* } \\
\hline & $A L$ & $C C$ & $S C$ & $L B$ & $A L$ & $C C$ & $S C$ & $L B$ \\
\hline 1 & 8 & 5 & 43 & 17 & 0 & 0 & 2 & -1 \\
\hline 2 & 94 & 75 & 91 & 33 & 81 & 55 & 83 & 3 \\
\hline 3 & 28 & 0 & 31 & -4 & 0 & 0 & 9 & 1 \\
\hline 4 & 33 & 25 & 56 & 27 & 0 & 0 & 2 & 1 \\
\hline 5 & 11 & 0 & 9 & 1 & 0 & 5 & 0 & 4 \\
\hline 6 & 25 & 0 & 61 & 3 & 0 & 0 & 7 & -1 \\
\hline 7 & 17 & 0 & 7 & 6 & 0 & 0 & 17 & 8 \\
\hline 8 & 33 & 25 & 43 & 11 & 42 & 25 & 65 & 24 \\
\hline 9 & 36 & 20 & 50 & 16 & 0 & 0 & 0 & 2 \\
\hline 10 & 78 & 55 & 87 & 54 & 3 & 0 & 0 & 5 \\
\hline 11 & 83 & 50 & 76 & NC & 0 & 0 & 4 & 0 \\
\hline 12 & 58 & 55 & 89 & -14 & 1 & 0 & 18 & -3 \\
\hline 13 & 22 & 0 & 4 & 2 & 0 & 0 & 4 & 1 \\
\hline
\end{tabular}

${ }^{\star} \mathrm{AL}=$ Albert lines; $\mathrm{CC}=$ circle cancellation; $\mathrm{SC}=$ star cancellation; $\mathrm{LB}=$ line bisection; $\mathrm{NC}=$ not conducted. after initial assessment. On initial testing patient 4 was unable to complete the chimeric faces task, and patient 12 was unable to complete the face matching task. All other patients completed both tasks. The mean (SD) period between initial testing and follow up was $54 \cdot 1$ $(4 \cdot 8)$ weeks. At this time, all patients had been discharged home or were in long-term care institutions. Interviews with patients and caregivers confirmed the absence of any subsequent strokes since the time of initial testing.

\section{Results}

CANCELLATION TASKS

Table 2 summarises the data from the cancellation tasks. Although the differences between the omissions made by the patients on initial and follow up testing are highly variable, it is clear that as a group these patients showed a substantial improvement in the 12 months between testing sessions. Separate one-way analyses of variance (ANOVAs) showed significant reductions in the percentage of omissions between initial and follow-up testing for each of the cancellation tasks (line cancellation, $F(1,12)=17 \cdot 284$, $\mathrm{p}<0.001$; circle cancellation, $F(1,12)=$ 7.622 , $\mathrm{p}<0.05$; star cancellation, $F(1,12)=$ $12 \cdot 136, \mathrm{p}<0.01$ ).

As targets in the line cancellation and star cancellation tasks are arranged into seven (roughly) vertical columns, it is possible to plot the percentage of omissions made by patients as a function of the horizontal location of each column on the page. ${ }^{12}$ Targets in the centre column were not considered as they were used by the examiner to show the task to the patient. Figure 2 shows the percentage of omissions made by patients in each testing session as a function of column position. In the initial testing session patients showed a linear decrease from left to right in the percentage of omissions on both cancellation tasks. The mean (SD) decrease in omissions from column to column was $14.3 \%$ (6.4) for the line cancellation and $14.9 \%(8.2)$ for the star cancellation task. Twelve $(92 \%)$ of the 13 patients showed this pattern of performance. In contrast, the relatively small number of omissions made by patients at 12 months tended to be more evenly distributed across the horizontal extent of the page. Only three patients $(23 \%)$ continued to show a spatially biased distribution of omissions in the follow-up assessment.

\section{LINE BISECTION}

Although the extent of line bisection errors decreased for most patients after 12 months, a one-way ANOVA, comparing mean errors from the initial testing session and those from the 12 month follow up, did not reach significance $(F(1,11)=2 \cdot 763, p>0 \cdot 05)$. Further analyses were conducted, however, in which the magnitude of bisection errors obtained from each testing session were considered separately. At the time of initial testing, the mean error on the line bisection task $(12.7 \mathrm{~mm})$ was significantly different from 
Figure 2 Mean (SE) percentage omissions on (A) Albert lines and (B) star cancellation task as a function of horizontal position of targets on page. = Initial testing

$\mathrm{O}=12$ month follow up.

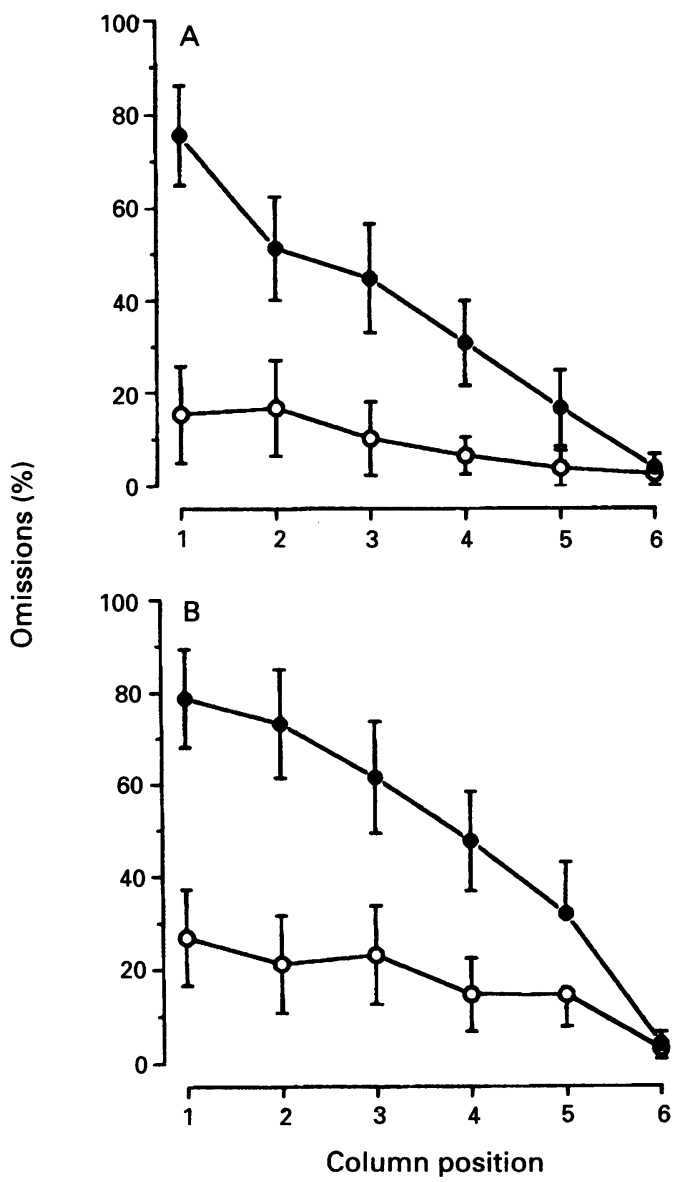

zero $(t(11)=2.381, \mathrm{p}<0.05)$. In contrast, reassessment after 12 months showed a substantial improvement in mean bisection error $(3.7 \mathrm{~mm})$, which was not significantly different from zero $(t(11)=1.803, p>0.05)$. It is therefore reasonable to conclude that after 12 months the patients' mean displacements were no longer significantly different from the "ideal" of zero error.

Figure 3 shows the mean bisection error plotted as a function of line length. For each testing session bisection errors for the group remained to the right of the true midpoint at all line lengths. Although patients' errors in

Figure 3 Mean (SE) bisection error on line bisection test as a function of line length $=$ initial testing; $O=12$ month follow up. the initial testing session showed a positively sloping linear relation with increasing line length, however, error magnitude remained relatively constant as a function of line length on reassessment at 12 months. The regression equations for patients' errors are: initial testing $=-11.315+(0.192 \times$ line length $)$, accounting for $95 \%$ of the variance; 12 months follow up $=-1.948+(0.043 \times$ line length) accounting for $66 \%$ of the variance.

\section{FACES TASKS}

A detailed analysis of patients' performances on initial testing (and that of healthy controls) is provided elsewhere. ${ }^{44}$ Briefly, controls show a significant leftward bias on the face matching and chimeric faces tasks-that is, they prefer to choose the left-left symmetrical composite (as viewed) as more closely resembling the original (asymmetrical) face, and tend to judge chimeras as looking "happier" when the smiling half-face is on the left side. In contrast, patients with left neglect showed a strong and significant rightward (reversed) bias. ${ }^{44}$

Figure 4 shows that the mean asymmetry scores for controls (from our previous study) and those for patients on initial testing and after 12 months. Patients continued to show a significant rightward bias after 12 months on both the face matching task $(t(12)=2 \cdot 404$, $\mathrm{p}<0.05)$ and the chimeric faces task $(t(12)=$ $4.474, p<0.001)$. On the face matching task the magnitude of this rightward bias after 12 months tended to be reduced in relation to the initial assessment, though this difference did not reach statistical significance $(F(1,11)$ $=3.962, \mathrm{p}=0.07)$. Similarly, on the chimeric faces task the magnitude of the rightward bias did not change significantly between initial and 12 month follow-up assessments $(F(1,11)$ $=0.796$, ns). Therefore, although performance on the cancellation and line bisection tasks generally approached normality a year after initial testing, the faces tasks showed almost no such recovery.

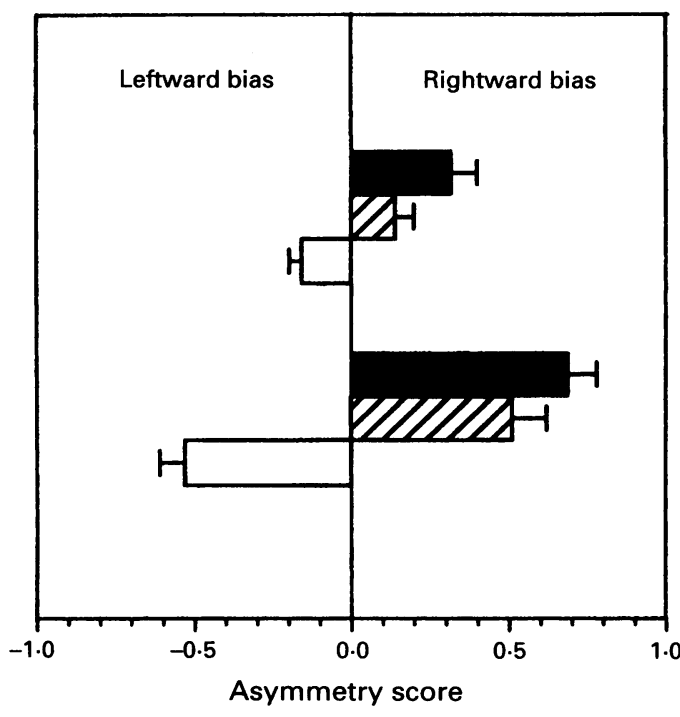

Figure 4 Mean (SD) asymmetry score on face matching (above) and chimeric faces tasks (below). Solid bars = Initial testing; shaded bars $=12$ month follow up; open bars $=$ normal controls. 
Figure 5 Mean percentage of abnormal scores on standard clinical (left) and faces (right) tasks. Solid bars = initial testing; shaded bars $=12$ months follow up. Patients were considered to have performed outside the normal range according to the following criteria: line cancellation more than four omissions; circle

cancellation more than one omission; star cancellation more than 15 omissions; $-3 \geqslant$ line bisection $\geqslant 3$ $\mathrm{mm}$; chimeric and face matching tasks $=$ positive asymmetry score.

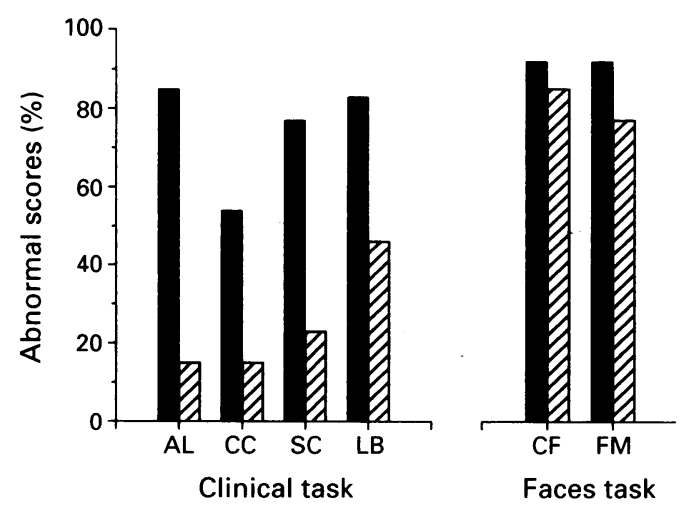

COMPARISON OF PERFORMANCES ON CLINICAL SCREENING TESTS AND FACES TASKS

The cancellation and line bisection tasks used in our study are widely used as measures of neglect in patients with unilateral cerebral damage. Some studies ${ }^{52}$ have provided normative data from which we obtained cutoff scores for distinguishing between normal and abnormal performances on these clinical measures. Figure 5 shows the percentages of patients classified as abnormal based on established cutoff scores at each testing session for the cancellation, line bisection, and faces tests. ${ }^{5} 4452$ On the cancellation tasks only a small percentage of patients continued to perform abnormally after 12 months: line cancellation $15 \%$, circle cancellation $15 \%$, star cancellation $23 \%$. On the line bisection test, relatively more patients $(46 \%)$ continued to perform abnormally after 12 months.

In marked contrast with the substantial reductions in impaired performances on these standard clinical tests, the percentage of patients who continued to show an abnormal perceptual bias on the faces tasks remained relatively high after 12 months. On the face matching and chimeric faces tasks, 77 and $85 \%$ of patients respectively continued to show a rightward bias. Thus a standard clinical evaluation incorporating only cancellation tasks would have classified most of the patients as "normal" after 12 months. By supplementing this battery with a line bisection test, slightly more than half of our patients would still have been classified as normal after 12 months. The addition of our faces tasks, however, allowed us to distinguish a substantial majority of patients as being outside the "normal" range, a finding which indicates the continued presence of an attentional bias which is no longer indexed by standard clinical tasks after 12 months.

\section{Discussion}

In the acute stages after right hemisphere damage patients with left unilateral neglect showed a strong and consistent ipsilesional (rightward) attentional bias, in addition to an impairment in directing attention toward the contralesional (left) side (see later). On initial testing patients showed a strong and consistent rightward bias on each of the faces tasks. ${ }^{44}$ These results confirm the presence of a pervasive attentional bias toward the ipsilesional side of visual stimuli in patients with right hemisphere damage and left neglect. On initial testing, patients also showed a relatively high percentage of omissions on all three cancellation tasks. Moreover, the percentage of omissions on line cancellation and star cancellation tasks varied systematically as a function of the horizontal location of targets on the page. This progressive deterioration in performance from right to left (which shows the difficulty patients have in redirecting attention toward the contralesional side) has been previously reported in patients with left neglect. ${ }^{12}$ It may reflect the continued functioning of intact left hemisphere attentional mechanisms, ${ }^{12} 18$ which may itself be enhanced by use of the right hand (left hemisphere), either directly through manual activity, or as a consequence of right-sided spatial cueing. ${ }^{12} 25$

After 12 months there were significant improvements on each of the cancellation tasks, with most patients performing within the normal range. In addition, the few omissions made by these patients were now more evenly distributed across the page, perhaps reflecting the residual effects of a generalised reduction in attentional capacity. ${ }^{35-37}$

Consistent with previous reports, ${ }^{53}$ most (though not all) patients initially showed a significant rightward error on the line bisection test. At this time there was a positive linear relation between the magnitude of bisection errors and line length, which is likely to be determined conjointly by patients' tendency to orient initially to the right end of the line and their subsequent difficulty in redirecting attention (leftward) toward the true midpoint. ${ }^{52} 54$ On initial testing, four patients (patients 3,5,12, and 13) showed either a normal performance on the line bisection test or a leftward bisection error, despite making many left-sided -omissions on the cancellation tasks. Similar observations have been made by others, ${ }^{41}$ though it is noteworthy that in a previous group study of right hemisphere damaged patients ${ }^{55}$ only those with frontal or subcortical lesions showed such a dissociation. Our four patients also had damage to these areas, suggesting that lesion location may be critical in determining performance on this test.

After 12 months the patient group continued to show a small (though non-significant) rightward bisection error. More than half of the patients now performed within the normal range. In addition, there was no longer a positive linear relation between bisection error and line length. It has previously been shown that left neglect patients can reduce or eliminate rightward bisection errors when their attention is drawn to the left end of the line immediately before placing their transection mark. ${ }^{52}{ }^{56}$ Regression equations obtained from the 12 month follow up were also comparable with those obtained previously under conditions in which patients were cued to the left end of the line before placing their transection mark. It is therefore likely that patients' accurate performance on the line bisection test after 12 months reflects restitution of their 
capacity to voluntarily direct attention (contralesionally) toward the left end of the line.

In contrast with these significant improvements, patients continued to show a strong bias toward the ipsilesional (right) side of stimuli in the two faces tasks. This attentional bias has been observed (on a different task) previously, ${ }^{32}$ though we are unaware of any study which has examined patients prospectively after 12 months. Karnath ${ }^{13}$ tested three patients with right hemisphere damage, two of whom had left neglect, on a tachistoscopic naming task. The patients were retested several months later and continued to show a right visual field bias on bilateral simultaneous stimulation. There was no evidence of an attentional bias on unilateral tachistoscopic presentation, however, nor on standard clinical tests of neglect and extinction. Gainotti et $a l^{32}$ examined the performances of patients with unilateral brain damage on an overlapping figures task. They found that whereas controls tended first to identify figures on the left of the composite diagram, patients with left neglect first identified figures on the right, suggesting an early orienting of attention to the ipsilesional side. Moreover, these workers suggested that right hemisphere lesioned patients without neglect were also more likely than controls to select right-sided figures first. To summarise, these data are consistent with our own findings that patients no longer showing classical signs of neglect (as indexed by the line bisection and cancellation tasks) continue to show an ipsilesional attentional bias on the faces tasks.

Previous workers have emphasised the importance of having "competing stimuli" to elicit the ipsilesional attentional bias. ${ }^{17}$ Thus when several stimuli are presented on either side of fixation ${ }^{1732}$ or when there is only a limited time to attend to a stimulus display, ${ }^{13}$ patients tend to show an ipsilesional bias. Using the two faces tasks we have shown that patients continue to exhibit a rightward attentional bias to visual stimuli presented in isolation (along the left-right dimension) and in free vision. The chimeric faces and face matching tasks provide a relatively "pure" measure of attentional bias (individual face stimuli in the two tasks subtend visual angles of $11^{\circ}$ and $8^{\circ}$ respectively) in the absence of any requirements for visual search. In addition to being easy to administer, these tasks also have the advantage of being psychometrically sound, with high split-half and test-retest reliability. ${ }^{44} 46$

Unlike the line bisection and cancellation tasks, the faces tests do not involve a directed motor response. Although it is possible that this difference may underlie the discrepancies in performance on the two types of task after 12 months, this seems unlikely. Several studies have found no difference between patients' performances on "perceptual" and "motor" versions of the same test. ${ }^{55}$ Moreover, our own informal observations suggest that patients can perform normally on other purely "perceptual" tasks, such as describing details of a complex scene. We believe that the residual ipsilesional bias observed here is most reliably indexed by tasks which involve only a single attentional fixation, without the need to serially reorient attention (by scanning or cancelling targets). We also suggest that the bias observed with the faces tasks reflects a general attentional bias, rather than a face-specific bias. This is supported by evidence from studies of normal subjects, indicating a similar (leftward) attentional bias for facial and nonfacial stimuli. ${ }^{57}$

With respect to the stimuli used in each test, it is clear that patients showed a stronger attentional bias on the chimeric faces task than on the face matching task. This was found on initial testing and in the 12 month follow-up assessment. In our previous study we suggested that this difference may be due to the increased difficulty involved in the face matching task, where subjects must make two separate comparisons between each symmetrical composite and the original face, and a further comparison between these two judgements. In the chimeric faces task only a single comparison between the two stimuli is required. It is also possible that the chimeric faces task and face matching task engage separate neural mechanisms, one responsible for processing facial emotion and the other for processing facial identity..$^{58}$

The question of whether a residual ipsilesional attentional bias is predictive of persistent "neglect-type" behaviours remains to be elucidated. Clearly, measures of functional abilities such as the Barthel test are not sufficient to index the often subtle, but nonetheless pervasive, deficits which may persist in the chronic stages of recovery. In the absence of a standardised scale for the functional assessment of spatial/attentional deficits, we can only rely on anecdotal evidence. On follow-up assessments, several of our patients (and their caregivers) reported a tendency to look rightward to any visual stimulus which entered the periphery of their visual field. One patient reported difficulties in watching television because his attention would spontaneously shift toward any movement visible from a window on his right. Another found that while conversing, his gaze would shift away from the person's face and toward some other location on the right. A small number of patients had also failed on-road driving tests because of "poor road positioning". We can only surmise that these problems arose under specific circumstances and in most cases did not interfere with patients' capacity for independent or semi-independent living.

Our findings are not only relevant for understanding the processes of natural recovery from neglect, but also have implications for attempts to facilitate such processes. For example, Pizzamiglio et al ${ }^{40}$ examined the performances of a small group of left neglect patients before and after specific retraining. They found significant improvements in visual scanning/exploration (as measured by reading and cancellation tasks), but only relatively small improvements on a task which purportedly measures perceptual bias (the 
Wundt-Jastrow illusion test). On the basis of our own findings of a strong, residual attentional bias in the absence of an impairment in directing attention to the contralesional side, we predict that such a bias is possibly the one component of neglect that does not readily recover and may not be amenable to rehabilitation.

We gratefully acknowledge the assistance of the administration and staff at the following institutions: Alfred Hospital, Austin Hospital, Hampton Rehabilitation Hospital, Heidelber Repatriation Hospital, Kingston Centre, Monash Medical Centre, and Royal Talbot Rehabilitation Hospital. We sincerely thank Vladimir Kohout for producing the photographic material. This study was supported by grants from the Australian Research Council and the Faculty of Education, Monash University.

1 Bisiach E, Vallar G. Hemineglect in humans. In: Boller F, Grafman J, eds. Handbook of neuropsychology. Vol. 1 Amsterdam: Elsevier, 1988:195-222.

2 Colombo A, De Renzi E, Faglioni P. The occurrence of visual neglect in patients with unilateral cerebral disease. visual neglect in patients
Cortex 1976;12:221-31.

3 Gainotti G, Messerli P, Tissot R. Qualitative analysis of unilateral spatial neglect in relation to laterality of cere-
bral lesions. $f$ Neurol Neurosurg Psychiatry 1972;35: bral lesions

4 Oxbury JM, Campbell DC, Oxbury SM. Unilateral spatial neglect and impairments of spatial analysis and visua perception. Brain 1974;97:551-64

5 Stone SP, Wilson B, Wroot A, et al. The assessment of visuo-spatial neglect after acute stroke. $\mathcal{f}$ Neurol Neurosurg Psychiatry 1991;54:345-50.

6 Ogden JA. Anterior-posterior interhemispheric differences in the loci of lesions producing visual hemineglect. Brain Cogn 1985;4:59-75.

7 Ogden JA. The "neglected" left hemisphere and its contribution to visuo-spatial neglect. In: Jeannerod $M$, ed. Neurophysiological and neuropsychological aspects of spatial neglect. Amsterdam: North-Holland, 1987:215-33.

8 Heilman KM, Watson RT, Valenstein E. Neglect and related disorders. In: Heilman KM, Valenstein E, eds. related disorders. In: Heilman KM, Valenstein E, eds. Clinical neuropsycholo

9 Mattingley JB, Bradshaw JL, Phillips JG. Reappraising unilateral neglect. Aust $\mathcal{F}$ Psychol 1992;44:163-9.

10 Mesulam M-M. Attention, confusional states, and neglect. In: Mesulam M-M, ed. Principles of behavioral neurology. Philadelphia: Davis, 1985:125-67.

11 Friedrich FJ, Margolin DI. Response time measures of hemi-inattention: a longitudinal case report. Neuropsychiatry, Neuropsychol Behav Neurol 1993;6:54-9.

12 Halligan PW, Burn JP, Marshall JC, Wade DT. Visuo-spatial neglect: qualitative differences and laterality of cerebral lesion. $\mathcal{F}$ Neurol Neurosurg Psychiatry 1992;55: 1060-8.

13 Karnath, H-O. Deficits of attention in acute and recovered hemi-neglect. Neuropsychologia 1988;20:27-45.

14 Stone SP, Patel P, Greenwood RJ, Halligan PW. Measuring visual neglect in acute stroke and predicting Measuring visual neglect in acute stroke and predicting Neurosurg Psychiatry 1992;55:431-6.

15 Zoccolotti P, Antonucci G, Judica A, Montenero P, Pizzamiglio L, Razzano C. Incidence and evolution of the hemineglect disorder in chronic patients with unilateral right brain damage. Int $\mathcal{f}$ Neurosci 1989;47: 209-16.

16 Bisiach E, Berti A. Dyschiria. An attempt at its systemic explanation. In: Jeannerod $M$, ed. Neurophysiological and neuropsychological aspects of spatial neglect. Amsterdam: North-Holland, 1987:183-201.

17 Gainotti G, D'Erme P, De Bonis C. Components of visual attention disrupted in unilateral neglect. In: Brown, JW, ed. Neuropsychology of visual perception. Hillsdale, NJ: Lawrence Erlbaum Associates, 1989:123-44.

18 Kinsbourne $M$. Mechanisms of unilateral neglect. In Jeannerod M, ed. Neurophysiological and neuropsychological aspects of spatial neglect. Amsterdam: North-Holland

19 Mesulam M-M. A cortical network for directed attention and unilateral neglect. Ann Neurol 1981;10:309-25.

20 Rizzolatti G, Camarda R. Neural circuits for spatial attention and unilateral neglect. In: Jeannerod $M$, ed. Neurophysiological and neuropsychological aspects of spatial neglect. Amsterdam: North-Holland, 1987:289-313.

21 Posner MI, Walker JA, Friedrich FA, Rafal RD. How do the parietal lobes direct covert attention? Neuropsychologia 1987;25:135-45.

22 Rizzolatti G, Gentilucci M, Matelli M. Selective spatial attention: one center, one circuit, or many cicuits? In: Posner MI, Marin OSM, eds. Attention and performance $X I$. Hillsdale, NJ: Lawrence Erlbaum Associates, 1985:251-65.

23 Posner MI, Walker JA, Friedrich FJ, Rafal RD. Effects of parietal injury on covert orienting of attention. $\mathcal{F}$ Neurosci 1984;4:1863-74.
24 Posner MI, Petersen SE. The attention system of the human brain. Annu Rev Neurosci 1990;13:25-42.

25 Halligan PW, Manning L, Marshall JC. Hemispheric activation vs spatio-motor cueing in visual neglect: a case study. Neuropsychologia 1991;29:165-76.

26 Hier DB, Mondlock J, Caplan LR. Recovery of behavioural abnormalities after right hemisphere stroke. Neurology 1983;33:345-50.

27 Wade DT, Wood VA, Hewer RL. Recovery of cognitive function soon after stroke; a study of visual neglect, attention span and verbal recall. $\mathcal{F}$ Neurol Neurosurg Psychiatry 1988;51:10-3.

28 Ládavas E, Menghini G, Umiltá C. A rehabilitation study of hemispatial neglect. Cogn Neuropsychol. In press.

29 D'Erme P, Robertson I, Bartolomeo P, Daniele A Gainotti G. Early rightwards orienting of attention on simple reaction time performance in patients with leftsimple reaction time performance in patients with
sided neglect. Neuropsychologia 1992;30:989-1000.

30 De Renzi E, Gentilini M, Faglioni P, Barbieri C. Attentional shift towards the rightmost stimuli in patients with left visual neglect. Cortex 1989;25 231-7.

31 Fisher $\dot{M}$. Left hemiplegia and motor impersistence. $\mathscr{f}$ Nerv Ment Dis 1956;123:201-18.

32 Gainotti G, D'Erme P, Bartolomeo P. Early orientation of attention toward the half-space ipsilateral to the lesion in patients with unilateral brain damage. $\mathcal{F}$ Neurol Neurosurg Psychiatry 1991;54:1082-9.

33 Campbell DC, Oxbury JM. Recovery from unilateral visuo-spatial neglect? Cortex 1976;12:303-12

34 Costa LD, Vaughn HG Jr, Howitz M, Ritter W. Patterns of behavioural deficits associated with visual spatial of behavioural deficits associated with

35 Robertson I. Anomalies in the laterality of omissions in unilateral left visual neglect: implications for an attentional theory of neglect. Neuropsychologia 1989;27: $157-65$.

36 Robertson IH. Digit span and visual neglect: a puzzling relationship. Neuropsychologia 1990;28:217-22.

37 Robertson I, Frasca R. Attentional load and visual neglect. Int $\mathcal{F}$ Neurosci 1992;52:45-56.

38 Petersen SE, Robinson DL, Currie JN. Influences of lesions of parietal cortex on visual spatial attention in humans. Exp Brain Res 1989;76:267-80.

39 Làdavas E. Selective spatial attention in patients with visual extinction. Brain 1990;113:1527-38.

40 Pizzamiglio L, Antonucci G, Judica A, Montenero $P$ Razzano C, Zoccolotti P. Cognitive rehabilitation of the hemineglect disorder in chronic patients with unilateral right brain damage. $f$ Clin Exp Neuropsychol 1992;14: right brain

41 Halligan PW, Marshall JC. Left visuo-spatial neglect: a meaningless entity? Cortex 1992;28:525-35.

42 Halligan PW, Marshall JC, Wade DT. Visuospatial neglect: underlying factors and test sensitivity. Lancet 1989;ii:908-10.

43 Kinsella G, Olver J, Ng K, Packer S, Stark R. Analysis of the syndrome of unilateral neglect. Cortex 1993;29. $135-40$.

44 Mattingley JB, Bradshaw JL, Phillips JG, Bradshaw JA. Reversed perceptual asymmetry for faces in left unilateral neglect. Brain Cogn 1993;23:145-65.

45 Gilbert C, Bakan P. Visual asymmetry in perception of faces. Neuropsychologia 1973;11:355-62.

46 Levy J, Heller W, Banich MT, Burton LA. Asymmetry of face perception in free viewing of chimeric faces. Brain Cogn 1983;2:404-19.

47 Patterson K, Bradshaw JL. Differential hemispheric mediation of nonverbal visual stimuli. $\mathcal{F}$ Exp Psychol [Hum Percept] 1975;1:246-52.

48 Mahoney FI, Barthel DW. Functional evaluation: the Barthel index. Maryland State Med f 1965;14:61-5.

49 Albert ML. A simple test of visual neglect. Neurology 1973;23:658-64

50 Ellis AW, Flude BM, Young AW. "Neglect dyslexia" and the early visual processing of letters in words and nonwords. Cognitive Neuropsychology 1987;4:439-64.

51 Wilson B, Cockburn J, Halligan PW. The behavioural inattention test. Titchfield: Thames Valley Test Company, 1987.

52 Mattingley JB, Pierson JM, Bradshaw JL, Phillips JG, Bradshaw JA. To see or not to see: the effects of visible and invisible cues on line bisection judgements in unilateral neglect. Neuropsychologia 1993;31:1201-15.

53 Schenkenberg T, Bradford DC, Ajax ET. Line bisection and unilateral visual neglect in patients with neurological and unilateral visual neglect in patients with

54 Nichelli P, Rinaldi M, Cubelli R. Selective spatial attention and length representation in normal subjects and in tion and length representation in normal subjects and in patients with

55 Binder J, Marshall R, Lazar R, Benjamin J, Mohr JP. Distinct syndromes of hemineglect. Arch Neurol 1992, 49:1187-94.

56 Riddoch JM, Humphreys GW. The effect of cueing on unilateral neglect. Neuropsychologia 1983;21:589-99.

57 Luh KE, Rueckert LM, Levy J. Perceptual asymmetries fo free viewing of several types of chimeric stimuli. Brain Cogn 1991;16:83-103.

58 Pizzamiglio L, Zoccolotti P, Mammucari A, Cesaroni R The independence of face identity and facial expression recognition mechanisms: relationship to sex and cognitive style. Brain Cogn 1983;2:176-88. 